\title{
EXACT SOLVABILITY OF TWO-DIMENSIONAL REAL SINGULAR MORSE POTENTIAL
}

\author{
M.V. Ioffe ${ }^{1}$, D.N. Nishnianidze ${ }^{1,2}$ \\ ${ }^{1}$ St.-Petersburg State University, 198504 St.-Petersburg, Russia \\ ${ }^{2}$ Kutaisi State University, 4600 Kutaisi, Georgia
}

November 3, 2018

\begin{abstract}
The supersymmetric approach in the form of second order intertwining relations is used to prove the exact solvability of two-dimensional Schrödinger equation with generalized two-dimensional Morse potential for $a_{0}=-1 / 2$. This two-parametric model is not amenable to conventional separation of variables, but it is completely integrable: the symmetry operator of fourth order in momenta exists. All bound state energies are found explicitly, and all corresponding wave functions are built analytically. By means of shape invariance property, the result is extended to the hierarchy of Morse models with arbitrary integer and half-integer values $a_{k}=-(k+1) / 2$.
\end{abstract}

PACS numbers: 03.65.-w, 03.65.Fd, 11.30.Pb

Introduction. - Very limited list of exactly solvable one-dimensional models is known up to now: harmonic oscillator, singular harmonic oscillator, Coulomb, Morse, Pöschl-Teller potentials, and some others [1]. As for complete solution of the Schrödinger equation in two-dimensional space, no regular approach is known. In practice, only the conventional separation of variables [2] provides the sole method to solve two-dimensional stationary quantum problems [3]. One of the fundamental problems of modern Quantum Mechanics is to enlarge as much as possible the variety of exactly solvable multi-dimensional $(d \geq 2)$ systems.

The supersymmetric approach has provided a powerful impulse for new development in analytical studies both in one-dimensional [6] and multi-dimensional [7] Quantum Mechanics. But to date, even among two-dimensional potentials, not amenable to standard separation of variables [8], very few results on partially solvable models were obtained. By means of supersymmetry, several two-dimensional models were found [9] - [12], for which a part of their 
spectra and wave functions were found analytically (partial solvability). It is necessary to stress that all constructed systems are completely integrable, since the symmetry operators of fourth order in momenta were built for them. Two specific supersymmetric techniques - the special SUSY-separation of variables and two-dimensional shape invariance - allowed to investigate two-dimensional singular generalizations of Morse [9] and Pöschl-Teller [11] potentials. The first method explores the most important constituent of SUSY algebra SUSY intertwining relations. The second method is the natural generalization of the onedimensional shape invariance approach [13], which provided an elegant algebraic algorithm for solution of a class of Schrödinger equations. In one-dimension, the class of shape invariant potentials coincides practically with the known variety of exactly solvable models [1].

Among all possible two-dimensional intertwining relations with supercharges of second order in derivatives, a subclass exists, where one of intertwined Hamiltonians is amenable to standard separation of variables due to specific choice of parameters of the model. Such situation was studied already for the complex two-dimensional Morse potential [15], which is free of any singularities. In the present Letter, we investigate the model, which is much more interesting physically - the real singular two-dimensional Morse potential with parameter value $a_{0}=-1 / 2$. Namely, we prove that, being completely integrable, this two-parametric model is exactly solvable as well, i.e. all its eigenvalues and eigenfunctions are known analytically. After that, we use two-dimensional shape invariance of the model to enlarge the class of exactly solvable two-dimensional systems to a hierarchy of Morse potentials with parameter values $a_{k}=-(k+1) / 2 ; k=1,2, \ldots$

Morse potential with $a_{0}=-1 / 2$. - Two-dimensional generalization of Morse potential $[9,10]$ is defined $[14]$ as:

$$
\widetilde{V}(\vec{x})=\frac{\alpha^{2} a(2 a-1)}{\sinh ^{2}\left(\frac{\alpha x_{-}}{2}\right)}+U\left(x_{1}\right)+U\left(x_{2}\right), \vec{x}=\left(x_{1}, x_{2}\right), x_{ \pm} \equiv x_{1} \pm x_{2},
$$

where $a$ is an arbitrary real parameter, and $U\left(x_{i}\right)$ are one-dimensional Morse potentials:

$$
U\left(x_{i}\right)=A\left(e^{-2 \alpha x_{i}}-2 e^{-\alpha x_{i}}\right) ; i=1,2 \quad \text { A }=\text { Const }>0 ; \alpha=\text { Const }>0,
$$

The Hamiltonian $\widetilde{H}(\vec{x})=-\Delta^{(2)}+\widetilde{V}(\vec{x})$ with potential (1) and the partner Hamiltonian 
$H(\vec{x})$ with potential:

$$
V(\vec{x})=\frac{\alpha^{2} a(2 a+1)}{\sinh ^{2}\left(\frac{\alpha x_{-}}{2}\right)}+U\left(x_{1}\right)+U\left(x_{2}\right) .
$$

are intertwined $[8]-[10]$

$$
\widetilde{H}(\vec{x}) Q^{+}=Q^{+} H(\vec{x}) ; \quad Q^{-} \widetilde{H}(\vec{x})=H(\vec{x}) Q^{-}
$$

by the second order supercharges:

$$
\begin{aligned}
Q^{ \pm} & =4 \partial_{+} \partial_{-} \pm 4 a \alpha \partial_{-} \pm 4 a \alpha \operatorname{coth} \frac{\alpha x_{-}}{2} \partial_{+}+4 a^{2} \alpha^{2} \operatorname{coth} \frac{\alpha x_{-}}{2}- \\
& -A\left[e^{-2 \alpha x_{1}}-2 e^{-\alpha x_{1}}-e^{-2 \alpha x_{2}}+2 e^{-\alpha x_{2}}\right] ; \quad \partial_{ \pm}=\frac{\partial}{\partial x_{ \pm}}
\end{aligned}
$$

One can notice that $\widetilde{V}$ and $V$ differ from each other by a coefficient in front of singular term only, this property being the origin of shape invariance of the model [16]. By construction, each intertwined Hamiltonian obeys the symmetry operator of fourth order in momenta $[9,10]$, which is expressed in terms of supercharges $Q^{ \pm}$. Specifically, the operator $\widetilde{R}=Q^{+} Q^{-}$ commutes with Hamiltonian $\widetilde{H}(\vec{x})$, and operator $R=Q^{-} Q^{+}$- with $H(\vec{x})$.

For the particular choice of parameter $a_{0}=-1 / 2$, the partner Hamiltonian (3) is simplified essentially - it allows the conventional separation of variables. Moreover, each of one-dimensional problems, obtained after separation, is exactly solvable one-dimensional Morse potential (2). The discrete spectrum of this model is well known [17]:

$$
\epsilon_{n}=-\alpha^{2} s_{n}^{2} ; \quad s_{n} \equiv \frac{\sqrt{A}}{\alpha}-n-\frac{1}{2}>0 ; \quad n=0,1,2, \ldots,
$$

and wave functions are expressed in terms of degenerate hypergeometric functions [18]:

$$
\eta_{n}\left(x_{i}\right)=\exp \left(-\frac{\xi_{i}}{2}\right)\left(\xi_{i}\right)^{s_{n}} F\left(-n, 2 s_{n}+1 ; \xi_{i}\right) ; \quad \xi_{i} \equiv \frac{2 \sqrt{A}}{\alpha} \exp \left(-\alpha x_{i}\right) .
$$

Due to separation of variables, the quantum problem with the Hamiltonian $H(\vec{x})$ from (3) is exactly solvable. Its energy eigenvalues are:

$$
E_{n, m}=E_{m, n}=\epsilon_{n}+\epsilon_{m},
$$

being two-fold degenerate for $n \neq m$. The corresponding eigenfunctions can be chosen as symmetric or (for $n \neq m$ ) antisymmetric combinations:

$$
\Psi_{E_{n, m}}^{S(A)}=\eta_{n}\left(x_{1}\right) \eta_{m}\left(x_{2}\right) \pm \eta_{m}\left(x_{1}\right) \eta_{n}\left(x_{2}\right) .
$$


Our aim here is to solve completely the quantum problem for $\widetilde{H}(\vec{x})$ with potential (1) for $a_{0}=-1 / 2$. The main tool are the SUSY intertwining relations (4), providing [10] connections between spectra and wave functions of partner Hamiltonians, which are almost isospectral (up to zero modes and singular properties of $Q^{ \pm}$).

In general, we may expect three kinds of levels of $\widetilde{H}(\vec{x})$.

(i). The levels, which coincide with (8). Their wave functions can be obtained from (9) by means of intertwining relations (4).

(ii). The levels, which were absent in the spectrum of $H(\vec{x})$, if some wave functions of $\widetilde{H}(\vec{x})$ are simultaneously the zero modes of the supercharge operator $Q^{-}$. Then the second intertwining relation in (4) would not give any partner state among bound states of $H(\vec{x})$.

(iii). The levels, which were also absent in the spectrum of $H(\vec{x})$, if some wave functions of $\widetilde{H}(\vec{x})$ become nonnormalizable functions after action of operators $Q^{-}$.

We will analyze these three classes of possible bound states of $\widetilde{H}(\vec{x})$ one after another.

(i). The first supersymmetric intertwining relation in (4) gives immediately the two-fold degenerate wave functions of $\widetilde{H}(\vec{x})$ with energies (8), as $\widetilde{\Psi}_{E_{n m}}=Q^{+} \Psi_{E_{n m}}$. Using the explicit formulas (5) for the supercharge $Q^{+}$and for one-dimensional Schrödinger equation (2), one can rewrite the symmetric (antisymmetric) wave functions in the form:

$$
\begin{gathered}
\widetilde{\Psi}_{E_{n, m}}^{S}=Q^{+} \Psi_{E_{n, m}}^{A}=\left(\epsilon_{m}-\epsilon_{n}\right) \Psi_{E_{n, m}}^{S}+D \Psi_{E_{n, m}}^{A}, \\
\widetilde{\Psi}_{E_{n, m}}^{A} \equiv Q^{+} \Psi_{E_{n, m}}^{S}=\left(\epsilon_{m}-\epsilon_{n}\right) \Psi_{E_{n, m}}^{A}+D \Psi_{E_{n, m}}^{S},
\end{gathered}
$$

where the differential operator $D$ is defined as:

$$
D=\frac{\alpha^{2}}{\xi_{2}-\xi_{1}}\left[\xi_{1}+\xi_{2}+2 \xi_{1} \xi_{2}\left(\partial_{\xi_{1}}+\partial_{\xi_{2}}\right)\right] .
$$

Since this operator is singular for $\xi_{1}=\xi_{2}$, normalizability of $\widetilde{\Psi}_{E_{n, m}}^{S, A}$ depends crucially on the behavior of $\Psi_{E_{n, m}}^{A, S}$ on the line $\xi_{1}=\xi_{2}$. The symmetric wave functions on this line are $\Psi_{E_{n, m}}^{S}\left(\xi_{1}, \xi_{2}=\xi_{1}\right) \sim \eta_{n}\left(\xi_{1}\right) \eta_{m}\left(\xi_{1}\right)$, and they have no zero multipliers $\left(\xi_{1}-\xi_{2}\right)$, which could compensate the singularity of operator $D$. Therefore, the wave functions $\widetilde{\Psi}_{E_{n, m}}^{A}=Q^{+} \Psi_{E_{n, m}}^{S}$ are certainly nonnormalizable, and they do not correspond to the physical bound states.

Vice versa, action of the operator $D$ in $(10)$ on the antisymmetric function $\Psi_{E_{n, m}}^{A}$ leads to nonsingular result. To observe this property, one has to use the explicit form (7) of one- 
dimensional eigenfunctions. After separation of exponential multiplier $\exp \left[-\left(\xi_{1}+\xi_{2}\right) / 2\right]$, the rest of function $\Psi_{E_{n, m}}^{A}$ is a polynomial in $\xi_{1}, \xi_{2}$. Due to antisymmetry, this polynomial vanishes for $\xi_{1}=\xi_{2}$, since it contains the multiplier $\left(\xi_{1}-\xi_{2}\right)$, compensating the singularity in $D$. Moreover, careful study shows that owing to the interplay between two terms in the r.h.s. of (10), the symmetric wave functions $\widetilde{\Psi}_{E_{n, m}}^{S} \sim\left(\xi_{1}-\xi_{2}\right)^{2}$ at the singular point $\xi_{1}=\xi_{2}$.

It is possible to investigate efficiently the normalizability of wave functions $\widetilde{\Psi}_{E_{n, m}}^{S}$ by the indirect algebraic method as well. One can check straightforwardly that for $a_{0}=-1 / 2$ the operator $R=Q^{-} Q^{+}$can be rewritten as:

$$
R=\left(h_{1}\left(x_{1}\right)-h_{2}\left(x_{2}\right)\right)^{2}+2 \alpha^{2}\left(h_{1}\left(x_{1}\right)+h_{2}\left(x_{2}\right)\right)+\alpha^{4},
$$

where $h_{i}\left(x_{i}\right) \equiv-\Delta_{i}+U\left(x_{i}\right)$ are one-dimensional Schrödinger operators with Morse potential (2). Acting by this operator on the antisymmetric function $\Psi_{E_{n, m}}^{A}$, one obtains:

$$
\begin{aligned}
R \Psi_{E_{n, m}}^{A}(\vec{x}) & =\left[\left(\epsilon_{n}-\epsilon_{m}\right)^{2}+2 \alpha^{2} E_{n, m}+\alpha^{4}\right] \Psi_{E_{n, m}}^{A}(\vec{x})= \\
& =\alpha^{4}\left[(n-m)^{2}-1\right]\left[\left(s_{n}+s_{m}\right)^{2}-1\right] \Psi_{E_{n, m}}^{A}(\vec{x}) \equiv r_{n, m} \Psi_{E_{n, m}}^{A}(\vec{x}) .
\end{aligned}
$$

This relation helps to find the norm of wave functions $\widetilde{\Psi}_{E_{n, m}}^{S}$ :

$$
\left\|\widetilde{\Psi}_{E_{n, m}}^{S}\right\|^{2}=\left\langle\Psi_{E_{n, m}}^{A}\left|Q^{-} Q^{+}\right| \Psi_{E_{n, m}}^{A}\right\rangle=r_{n, m}\left\|\Psi_{E_{n, m}}^{A}\right\|^{2}
$$

where we have used that $Q^{ \pm}$are mutually conjugate on the considered space. We stress that off-integral terms in (15) at the singular point $x_{1}=x_{2}$ disappear due to vanishing behavior of normalizable wave functions $\widetilde{\Psi}^{S}(\vec{x})$, mentioned above (see below Eqs.(17),(18) and nearby).

By definition, $\Psi_{E_{n, m}}^{A}$ in (15) are zero identically for $m=n$. In its turn, the wave functions $\Psi_{E_{n, n \pm 1}}^{A}$ are annihilated [15] by the action of $Q^{+}$, since the corresponding $r_{n, m}$ in (14) vanish for $m=n \pm 1$. It follows from (14), that the norms of all other $\widetilde{\Psi}_{E_{n, m}}^{S}$ (with $|n-m| \geq 2$ ) are finite and positive. Therefore, the first class of levels of $\widetilde{H}(\vec{x})$ consists of nondegenerate energy levels (8) with normalizable symmetric wave functions $\widetilde{\Psi}_{E_{n m}}^{S}$ from (10) with $|n-m| \geq 2$.

(ii). This class of possible bound states of the Hamiltonian $\widetilde{H}(\vec{x})$ with $a=-1 / 2$ consists of the normalizable zero modes of the supercharge $Q^{-}$from (5). In [9] the variety of normalizable zero modes of the Hermitian conjugated supercharge $Q^{+}$was studied in detail 
for arbitrary values of $a$. In particular, it was shown that these zero modes are normalizable, and no fall to the center occurs for the specific range for values of $a$. According to expressions (5), $Q^{-}$can be obtained from $Q^{+}$by replacing $a \rightarrow-a$. Therefore, completely analogous analysis of zero modes of $Q^{-}$will lead to the analogous, but positive, interval for $a$ :

$$
a \in\left(\frac{1}{4}+\frac{1}{4 \sqrt{2}},+\infty\right)
$$

which, for certain, does not contain the value $a_{0}=-1 / 2$. This means that no normalizable bound states of this class exist for $\widetilde{H}$.

(iii). We have to study an opportunity that operator $Q^{-}$destroys normalizability of some eigenfunctions of $\widetilde{H}$. It could occur due to singular character of $Q^{-}$at $x_{1}=x_{2}$. It is convenient to choose the polar coordinates $\xi, \varphi$ in the vicinity of this line in the plane $\left(\xi_{1}, \xi_{2}\right)$ :

$$
\xi_{1}=\xi \cos \varphi ; \quad \xi_{2}=\xi \sin \varphi ; \varphi \equiv \pi / 4-\theta ; \theta \sim 0 .
$$

In these variables the potential $\widetilde{V}(\vec{x})$ reads:

$$
\widetilde{V}=\alpha^{2}\left[\frac{\cos 2 \theta}{\sin ^{2} \theta}+\frac{1}{4} \xi^{2}-\left(s_{1}+\frac{3}{2}\right) \sqrt{2} \xi \cos \theta\right] \sim \alpha^{2} \frac{1}{\theta^{2}} ; \quad \theta \sim 0
$$

Thus, the Hamiltonian $\widetilde{H}(\vec{x})$ effectively acts as:

$$
\widetilde{H}=-\Delta^{(2)}+\widetilde{V}(\vec{x}) \sim-\alpha^{2}\left[\frac{1}{2} \partial_{\theta}^{2}-\frac{1}{\theta^{2}}\right] ; \quad \theta \sim 0,
$$

and two kinds of behavior of its eigenfunctions are possible:

$$
\widetilde{\Psi} \sim \theta^{2} \quad \text { or } \quad \widetilde{\Psi} \sim \theta^{-1} .
$$

Only the first one is normalizable at $\theta \sim 0$ with the measure $d x_{1} d x_{2}=d \xi d \theta / \xi \cos 2 \theta$.

In the same vicinity, the supercharge operator $Q^{-}$acts as:

$$
Q^{-} \sim \alpha^{2}\left(\xi \partial_{\xi}-1\right)\left(\partial_{\theta}+\frac{1}{\theta}\right) .
$$

This operator is not able to transform normalizable wave function of (18) to nonnormalizable. Therefore, the third class of possible wave functions $\widetilde{\Psi}$ of $\widetilde{H}(\vec{x})$ does not exist too.

Summing up the above analysis, we found that the spectrum of two-dimensional Hamiltonian $\widetilde{H}(\vec{x})$ with $a_{0}=-1 / 2$, which is not amenable to separation of variables, consists only 
of the bound states with energies (8) for $|n-m|>1$. This spectrum is bounded from above by the condition of positivity of $s_{n}, s_{m}$ in (6): $n, m<\sqrt{A} / \alpha-1 / 2$. The corresponding wave functions are obtained analytically, according to (7), (9), and (10).

Hierarchy of Morse potentials with $a_{k}=-(k+1) / 2$. - In what follows we denote the exactly solvable Hamiltonians $H(\vec{x}), \widetilde{H}(\vec{x})$ investigated above, as $H\left(\vec{x} ; a_{0}\right), \widetilde{H}\left(\vec{x} ; a_{0}\right)$. The motivation is that we will consider the Hamiltonians $H\left(\vec{x} ; a_{k}\right), \widetilde{H}\left(\vec{x} ; a_{k}\right)$ with arbitrary negative integer and half-integer values $a=a_{k}=-(k+1) / 2 ; k=0,1,2, \ldots$ We will prove that all these Hamiltonians are also exactly solvable.

The important property is that due to identity $a_{k-1}\left(2 a_{k-1}-1\right)=a_{k}\left(2 a_{k}+1\right)$ :

$$
\widetilde{H}\left(\vec{x} ; a_{k-1}\right)=H\left(\vec{x} ; a_{k}\right) ; k=1,2, \ldots
$$

This means that the following chain (hierarchy) of Hamiltonians can be built:

$$
H\left(\vec{x} ; a_{0}\right) \div \widetilde{H}\left(\vec{x} ; a_{0}\right)=H\left(\vec{x} ; a_{1}\right) \div \widetilde{H}\left(\vec{x} ; a_{1}\right)=\ldots \div \widetilde{H}\left(\vec{x} ; a_{k-1}\right)=H\left(\vec{x} ; a_{k}\right) \div \widetilde{H}\left(\vec{x} ; a_{k}\right)
$$

where the sign $\div$ between two Hamiltonians denotes their intertwining by $Q^{ \pm}\left(a_{i}\right)$.

Since the Hamiltonian $\widetilde{H}\left(\vec{x} ; a_{0}\right)$ (and therefore $H\left(\vec{x} ; a_{1}\right)$ ) was shown to be exactly solvable, we can use the intertwining relations from $(20)$ to prove exact solvability of $\widetilde{H}\left(\vec{x} ; a_{1}\right)$ too. Considering this procedure for the general length of the chain (20), we will illustrate results for the first section $k=1$ of the chain, with the Hamiltonian under study $\widetilde{H}\left(\vec{x} ; a_{1}=-1\right)$.

It was shown above, that bound states of the Hamiltonian $\widetilde{H}\left(\vec{x} ; a_{0}\right)=H\left(\vec{x} ; a_{1}\right)$ are described by the symmetric functions $\widetilde{\Psi}_{E_{m, n}}^{S}\left(\vec{x} ; a_{0}\right) \equiv \Psi_{E_{m, n}}^{S}\left(\vec{x} ; a_{1}\right)$ of $(10)$ and energy eigenvalues (8) with $|n-m|>1$. Therefore, the functions

$$
\widetilde{\Psi}_{E_{n, m}}^{A}\left(\vec{x} ; a_{1}\right)=Q^{+}\left(a_{1}\right) \Psi_{E_{n, m}}^{S}\left(\vec{x} ; a_{1}\right)=Q^{+}\left(a_{1}\right) Q^{+}\left(a_{0}\right) \Psi_{E_{n, m}}^{A}\left(\vec{x} ; a_{0}\right) ;|n-m|>1
$$

(if they are normalizable) are the wave functions of the partner Hamiltonian $\widetilde{H}\left(\vec{x} ; a_{1}\right)$. The only exclusion concerns possible zero modes of $Q^{+}\left(a_{1}\right)$. It was shown in [9], that the wave functions of $H\left(\vec{x} ; a_{k}\right)$ may coincide with zero modes of supercharge $Q^{+}\left(a_{k}\right)$ for energy levels:

$$
E_{n, n+k+1}=-2 \alpha^{2}\left(2 a_{k} s_{n}+s_{n}^{2}\right)-4 \alpha^{2} a_{k}^{2}=-\alpha^{2}\left(s_{n}^{2}+s_{n+k+1}^{2}\right) .
$$


In particular for $k=1$, operator $Q^{+}\left(a_{1}\right)$, acting on the wave functions $\Psi_{E_{n, n \pm 2}}\left(\vec{x} ; a_{1}\right)$ of $H\left(\vec{x} ; a_{1}\right)$, may annihilate them, not admitting to the spectrum of $\widetilde{H}\left(\vec{x} ; a_{1}\right)$.

Analogously in the general case, up to zero modes of $Q^{+}$the functions

$$
\widetilde{\Psi}_{E_{n, m}}\left(\vec{x} ; a_{k}\right)=Q^{+}\left(a_{k}\right) \Psi_{E_{n, m}}\left(\vec{x} ; a_{k}\right)=Q^{+}\left(a_{k}\right) Q^{+}\left(a_{k-1}\right) \ldots Q^{+}\left(a_{0}\right) \Psi_{E_{n, m}}^{A}\left(\vec{x} ; a_{0}\right)
$$

(again, if normalizable) are the wave functions of $\widetilde{H}\left(\vec{x} ; a_{k}\right)$ with energies $E_{n, m}=-\alpha^{2}\left(s_{n}^{2}+s_{m}^{2}\right)$. The symmetries of wave functions (22) alternate and depend on the length of chain (20).

In order to control the situation with possible zero modes of $Q^{+}$and normalizability of the states (22), we will use the following algebraic trick. For general value of $a_{k}$, the identity between Hamiltonians $H\left(\vec{x} ; a_{k}\right)=\widetilde{H}\left(\vec{x} ; a_{k-1}\right)$ leads to identity (up to a function of the Hamiltonian itself) between their symmetry operators $R\left(a_{k}\right)$ and $\widetilde{R}\left(a_{k-1}\right)$. This relation can be derived straightforwardly by algebraic manipulations with result:

$$
Q^{-}\left(a_{k}\right) Q^{+}\left(a_{k}\right)=Q^{+}\left(a_{k-1}\right) Q^{-}\left(a_{k-1}\right)+\alpha^{2}(2 k+1)\left[2 \widetilde{H}\left(\vec{x} ; a_{k-1}\right)+\alpha^{2}\left(2 k^{2}+2 k+1\right)\right] ;
$$

In the case $k=1$, Eq.(23) can be used to transform the norm of the function (21):

$$
\begin{aligned}
& \left\|\widetilde{\Psi}_{E_{n, m}}\left(\vec{x} ; a_{1}\right)\right\|^{2}=\left\langle\Psi_{E_{n, m}}^{A}\left(\vec{x} ; a_{0}\right)\left|Q^{-}\left(a_{0}\right) Q^{-}\left(a_{1}\right) Q^{+}\left(a_{1}\right) Q^{+}\left(a_{0}\right)\right| \Psi_{E_{n, m}}^{A}\left(\vec{x} ; a_{0}\right)\right\rangle=r_{n, m} \quad(24) \\
& \left(r_{n, m}+6 \alpha^{2} E_{n, m}+15 \alpha^{4}\right)\left\|\Psi_{n, m}^{A}\left(a_{0}\right)\right\|^{2}=\alpha^{4} r_{n, m}\left[(m-n)^{2}-4\right]\left[\left(s_{n}+s_{m}\right)^{2}-4\right]\left\|\Psi_{n, m}^{A}\left(a_{0}\right)\right\|^{2},
\end{aligned}
$$

where definitions (14) for $r_{n, m}$ were explored. From Eq.(24), the (real) functions $\widetilde{\Psi}_{n, n \pm 2}\left(\vec{x} ; a_{1}\right)$ vanish identically for $m=n \pm 2$, being zero modes of $Q^{+}\left(a_{1}\right)$. Therefore, energies $E_{n, n+2}$ are absent in the spectrum of $\widetilde{H}\left(\vec{x} ; a_{1}\right)$. Vise versa, due to the same Eq.(24) the norms of all other $\widetilde{\Psi}_{n, m}\left(\vec{x} ; a_{1}\right)$ with $|n-m|>2$ are positive and finite.

This analysis can be generalized to arbitrary integer values of $k$. The proof is performed step by step, by using Eq.(23) for indices $k, k-1, \ldots, 1,0$ in calculation of the norm:

$$
\left\|\widetilde{\Psi}_{E_{n, m}}\left(\vec{x} ; a_{k}\right)\right\|^{2}=\left\langle\Psi_{E_{n, m}}^{A}\left(\vec{x} ; a_{0}\right)\left|Q^{-}\left(a_{0}\right) \ldots Q^{-}\left(a_{k}\right) Q^{+}\left(a_{k}\right) \ldots Q^{+}\left(a_{0}\right)\right| \Psi_{E_{n, m}}^{A}\left(\vec{x} ; a_{0}\right)\right\rangle .
$$

After some algebra, one obtains that

$$
\begin{aligned}
\left\|\widetilde{\Psi}_{E_{n, m}}\left(\vec{x} ; a_{k}\right)\right\|^{2} & =\left\langle\Psi_{E_{n, m}}^{A}\left(\vec{x} ; a_{0}\right)\right| R\left(a_{0}\right)\left(R\left(a_{0}\right)+\Gamma_{0}\right)\left(R\left(a_{0}\right)+\Gamma_{0}+\Gamma_{1}\right) \ldots \\
& \ldots\left(R\left(a_{0}\right)+\Gamma_{0}+\ldots+\Gamma_{k-1}\right)\left|\Psi_{E_{n, m}}^{A}\left(\vec{x} ; a_{0}\right)\right\rangle ; \\
\Gamma_{l} \quad & \equiv \alpha^{2}(2 l+1)\left[2 H\left(a_{0}\right)+\alpha^{2}\left(2 l^{2}+2 l+1\right)\right] .
\end{aligned}
$$


Inside the matrix element $(26), R\left(a_{0}\right)$ can be replaced by its eigenvalues (14), and sums $\left(\Gamma_{0}+\Gamma_{1}+\ldots+\Gamma_{i}\right)$ by:

$$
\sum_{l=1}^{l=i}\left[2 \alpha^{2}(2 l+1) E_{n, m}+\alpha^{4}\left((l+1)^{4}-l^{4}\right)\right]=\alpha^{4}\left[-2\left(s_{n}^{2}+s_{m}^{2}\right) i(i+2)+(i+1)^{4}-1\right] .
$$

Thus, the norm (25) can be factorized:

$$
\left\|\widetilde{\Psi}_{E_{n, m}}\left(\vec{x} ; a_{k}\right)\right\|^{2}=\alpha^{4 k}\left\|\Psi_{E_{n, m}}^{A}\left(\vec{x} ; a_{0}\right)\right\|^{2} r_{n, m} \prod_{i=1}^{k}\left[(n-m)^{2}-(i+1)^{2}\right]\left[\left(s_{n}+s_{m}\right)^{2}-(i+1)^{2}\right] .
$$

One can conclude that the norm vanishes for $|n-m| \leq(k+1)$, and it is finite positive for other $n, m$. This means that the spectrum of $\widetilde{H}\left(\vec{x} ; a_{k}\right)$ includes all energy levels $E_{n, m}$ of $H\left(\vec{x} ; a_{0}\right)$ with $|n-m|>(k+1)$ only. All others disappear due to zero modes of $Q^{+}\left(a_{i}\right)$.

Similarly to the analysis (ii) above, no normalizable wave functions of $\widetilde{H}\left(\vec{x} ; a_{k}\right)$ can be annihilated by the operator $Q^{-}\left(a_{k}\right)$, since the values $a_{k}=-(k+1) / 2$ lie again outside of the interval (16), permitted for their normalizability. The class (iii) of possible bound states of $\widetilde{H}\left(\vec{x} ; a_{k}\right)$ is also empty. To prove this fact, one has to consider the behavior of operators $\widetilde{H}, Q^{-}$and normalizable wave functions $\widetilde{\Psi}$ at the point of singularity $\theta \sim 0$ :

$$
\begin{aligned}
& \widetilde{H}\left(\vec{x} ; a_{k}\right) \sim-\frac{\alpha^{2}}{2}\left[\partial_{\theta}^{2}-\frac{k+1}{\theta^{2}}\right] ; \quad \widetilde{\Psi}_{E_{n, m}}\left(\vec{x} ; a_{k}\right) \sim \theta^{k+2} \\
& Q^{-}\left(a_{k}\right) \sim \alpha^{2}\left(\xi \partial_{\xi}-(k+1)\right)\left(\partial_{\theta}+\frac{k+1}{\theta}\right) .
\end{aligned}
$$

Similar to the case $k=0$, operators $Q^{-}\left(a_{k}\right)$ can not destroy normalizability of $\widetilde{\Psi}_{E_{n, m}}\left(\vec{x} ; a_{k}\right)$.

Summarizing the obtained results, the spectra of Hamiltonians $\widetilde{H}\left(\vec{x} ; a_{k}\right)$ are not degenerate. They consist of the bound states with energies $E_{n, m}$, given by (8) with indices $|n-m|>k+2$. Their wave functions $\widetilde{\Psi}_{E_{n, m}}\left(\vec{x} ; a_{k}\right)$ are given by Eq.(22) in terms of degenerate (confluent) hypergeometric functions. Like in the case $a_{0}=-1 / 2$, the discrete spectra are bounded from above by the conditions (6): $n, m<\sqrt{A} / \alpha-1 / 2$.

The work was partially supported by grants RFFI 06-01-00186-a, RNP 2.1.1.1112.

\section{References}

[1] L. Infeld and T.E. Hull, Rev.Mod.Phys. 23, 21 (1951). 
[2] W.Miller Jr., Symmetry and Separation of Variables (Addison-Wesley, London, 1977).

[3] We must recall also the class of exactly solvable models of $N$ particles on a line, so called Calogero and Calogero-like models [4]. In a particular case of $N=3$, such models can be interpreted as one-particle models on the plane [5].

[4] F. Calogero and C. Marchioro, J.Math.Phys. 14182 (1973); M. Olshanetsky and A.M. Perelomov, Phys. Rep. 94313 (1983).

[5] F.Cannata and M.V.Ioffe, J.Phys.A:Math.Gen. 341129 (2001); M.V.Ioffe and A.I.Neelov, J.Phys.A:Math.Gen. 357613 (2002)

[6] E.Witten, Nucl.Phys.B 188513 (1981); F.Cooper, A. Khare and U. Sukhatme, Phys.Rep. 251268 (1995); G.Junker, Supersymmetrical Methods in Quantum and Statistical Physics (Springer, Berlin, 1996); B.K.Bagchi, Supersymmetry in Quantum and Classical Mechanics (Chapman, Boca Raton, 2001).

[7] A.A.Andrianov, N.V.Borisov, M.I.Eides and M.V.Ioffe, Phys.Lett.A 109143 (1985).

[8] A.Andrianov, M.Ioffe and D.Nishnianidze, Phys.Lett.A 201103 (1995); Theor. and Math.Phys. 1041129 (1995); solv-int/9605007; J.Phys.A:Math.Gen. 324641 (1999).

[9] F.Cannata, M.V.Ioffe and D.N.Nishnianidze, J.Phys.A:Math.Gen. 351389 (2002).

[10] M.V.Ioffe, J.Phys.A:Math.Gen. 3710363 (2004).

[11] M.V.Ioffe and P.A.Valinevich, J.Phys.A:Math.Gen. 382497 (2005); M. V. Ioffe, J. Mateos Guilarte and P. A. Valinevich, Ann.Phys. 3212552 (2006); hep-th/0706.1344.

[12] M.V.Ioffe, J.Negro, L.M.Nieto and D.N.Nishnianidze, J.Phys.A:Math.Gen. 399297 (2006).

[13] L.E. Gendenshtein, JETP Lett. 38356 (1983)

[14] This definition coincides with the definitions in [9], but up to an additive constant. 
[15] F.Cannata, M.V.Ioffe and D.N.Nishnianidze, Theor.Math.Phys. 148960 (2006); hepth/0704.2219.

[16] F.Cannata, M.V.Ioffe and D.N.Nishnianidze, Phys.Lett.A 34031 (2005).

[17] L.Landau, E.Lifshitz, Quantum Mechanics (Pergamon, London, 1965) (Sect.23).

[18] H.Bateman, E.Erdelyi, Higher Transcendental Functions (v.1) (McGraw-Hill,NY, 1953). 